\title{
A Seroepidemiological Survey of Toxoplasma gondii and Chlamydia Infection in Chickens, Ducks, and Geese in Jilin Province, Northeastern China
}

\author{
Ming-Han Li, ${ }^{1, *}$ Bin-Tong Yang, ${ }^{1,2, *}$ Zhi-Wei Yin, ${ }^{1}$ Wei Wang, ${ }^{3}$ Quan Zhao, ${ }^{1}$ and Jing Jiang ${ }^{2}$
}

\begin{abstract}
Toxoplasma gondii and Chlamydia are common protozoan pathogens, which can pose health risks to people around the world and cause considerable economic losses. Although information on the seroprevalence of $T$. gondii and Chlamydia in chickens can be found in China, knowledge on the infection of these two diseases in rural chickens, ducks, and geese is limited. Therefore, blood samples from 377 chickens, 268 ducks, and 379 geese were taken from rural areas in seven cities in northeastern China, and their seropositivity was analyzed by the indirect hemagglutination antibody (IHA) test with a cutoff titer of 1:64. The overall seroprevalence of T. gondii and Chlamydia was $10.6 \%$ and $21.0 \%$, respectively. Moreover, the free-range poultry $(20.75 \%$ and $30.55 \%)$ have significantly higher seroprevalences than caged poultry $(5.47 \%$ and $15.66 \%)(p<0.001)$. Moreover, the seroprevalence of $T$. gondii in older and younger poultry was found to be $5.48 \%$ and $12.89 \%$, and those in Chlamydia were $23.53 \%$ and $15.16 \%$, respectively. There were significant differences between different ages $(p<0.05)$. But regarding the prevalence of both, it is not clear in this way which one is the prevalence for younger and older for each specific pathogen. Logistic regression analysis suggested that geography were considered as a risk factor for $T$. gondii infection in this study. Our study is the first report on the seroprevalence of $T$. gondii and Chlamydia in poultry in rural areas of Jilin Province, China. These data reflect that $T$. gondii and Chlamydia are widespread in poultry in northeastern China, leading to a risk of infection in human health. Therefore, the prevention and control of Toxoplasma and Chlamydia infection in poultry, other animals, and humans should be strengthened.
\end{abstract}

Keywords: serology, Toxoplasma gondii, Chlamydia, poultry, indirect hemagglutination test, China

\section{Introduction}

IN ChIna, WITH THE RAPID development of the poultry industry and the number of poultry raised by farmers is increasing. Eggs and poultry meat are not only the important source of animal protein ingested by humans, but also can spread a variety of pathogens such as Toxoplasma gondii and Chlamydia (Hill and Dubey 2016).

Toxoplasmosis is a zoonosis caused by $T$. gondii (Zhang et al. 2015). It is widely parasitic in human and animal nucleated cells (Kang et al. 2016). Most people are carriers of T. gondii, accounting for one third of the world's population (Weiss and Dubey 2009, Zhang et al. 2019). Infected patients usually have asymptomatic infections or recurrent choroidal retinitis, and severe cases can lead to death of patients with weakened immune function. Congenital infections of $T$. gondii may cause miscarriages, stillbirths, or deformities in the first trimester of pregnancy (Jones et al. 2001, Elmore et al. 2010). Chlamydiosis is a group of infectious diseases caused by various Chlamydia infections, with 11 species currently described (Sachse et al. 2015). It can be transmitted to humans by poultry directly or indirectly contacting feces of Chlamydia psittaci. The typical clinical manifestations of Chlamydia infection in humans or animals are high fever, dyspnea, encephalitis, myelitis, and pneumonia (Wang et al. 2012, Qin et al. 2014). The epidemic characteristics of these pathogens showed widespread and diversified transmission sources making it difficult to control effectively and seriously affecting the economic benefits of the farmers (Dubey 2010, Zhang et al. 2019).

\footnotetext{
${ }^{1}$ Department of Parasitology, College of Animal Science and Technology, Jilin Agricultural University, Changchun, P.R. China.

${ }^{2}$ Department of Parasitology, College of Life Science, Changchun Sci-Tech University, Shuangyang, P.R. China.

${ }^{3}$ Department of Parasitology, College of Life Science and Biotechnology, Heilongjiang Bayi Agricultural University, Daqing, P.R. China.

*These authors contributed equally to this work.
} 
In the past 5 years, Toxoplasma and Chlamydia infections have been reported around the world (Krawiec et al. 2015, Sachse et al. 2015, Li et al. 2016, 2017), but there was no information on the two pathogens in poultry in northeastern China, especially in the chickens, ducks, and geese raised in the countryside. We described the serological status of T. gondii and Chlamydia infection in some rural areas of Jilin province. Also, the prevalence of parasitic infections was a good indication of the presence of parasitic oocysts near human living areas. By clarifying, the epidemic risk factors can provide a more reasonable and effective way to control the transmission of these pathogens in China.

\section{Materials and Methods}

\section{Ethics statement}

All operations were handled in strict accordance with the Good Animal Practice requirements of the Animal Ethics Procedures and Guidelines of the People's Republic of China. This study was approved by the Animal Ethics Committee of Jilin Agricultural University.

\section{Sampling and sera preparation}

A total of 1024 poultry blood samples (including chickens, ducks, and geese) were collected from free-range and intensive poultry farms in rural areas from Siping City $\left(n=142,43^{\circ} 01^{\prime}\right.$ to $44^{\circ} 52^{\prime} \mathrm{N}, 124^{\circ} 13^{\prime}$ to $125^{\circ} 58^{\prime} \mathrm{E}$ ), Yanji City $\left(n=131,42^{\circ} 50^{\prime}\right.$ to $43^{\circ} 23^{\prime} \mathrm{N}, 129^{\circ} 01^{\prime}$ to $\left.129^{\circ} 48^{\prime} \mathrm{E}\right)$, Jilin City $\left(n=174,42^{\circ} 31^{\prime}\right.$ to $44^{\circ} 40^{\prime} \mathrm{N}, 125^{\circ} 40^{\prime}$ to $\left.127^{\circ} 56^{\prime} \mathrm{E}\right)$, Baishan City $\left(n=115,41^{\circ} 21^{\prime}\right.$ to $42^{\circ} 49^{\prime} \mathrm{N}, 126^{\circ} 07^{\prime}$ to $\left.128^{\circ} 18^{\prime} \mathrm{E}\right)$, Tonghua City $(n=84$, $40^{\circ} 52^{\prime}$ to $43^{\circ} 03^{\prime} \mathrm{N}, 125^{\circ} 10^{\prime}$ to $126^{\circ} 44^{\prime} \mathrm{E}$ ), Changchun City ( $n=223,43^{\circ} 05^{\prime}$ to $45^{\circ} 15^{\prime} \mathrm{N}, 124^{\circ} 18^{\prime}$ to $127^{\circ} 05^{\prime} \mathrm{E}$ ), and Fusong County ( $n=155,41^{\circ} 42^{\prime}$ to $42^{\circ} 49^{\prime} \mathrm{N}, 127^{\circ} 01^{\prime}$ to $128^{\circ} 06^{\prime} \mathrm{E}$ ) of Jilin Province. Detailed information on all samples (e.g., region, age, gender, and so on) is provided by the owner of the farm and the number is recorded in Table 1. The blood sample was placed in a $37^{\circ} \mathrm{C}$ incubator for $2-4 \mathrm{~h}$ and put in a $4^{\circ} \mathrm{C}$ refrigerator for about $2 \mathrm{~h}$ before using, and then performed in a centrifuge at $1500 \times g$ for $10 \mathrm{~min}$. Generated blood sample layered, in which the upper liquid is the serum. The supernatant was transferred to an autoclaved $1.5 \mathrm{~mL}$ Eppendorf (EP) tube after autoclaving under aseptic conditions and stored at $-20^{\circ} \mathrm{C}$.

\section{Serological examination}

Antibodies against $T$. gondii and Chlamydia spp. from serum samples were detected by an indirect hemagglutination antibody (IHA) test using a commercially available kit obtained from Lanzhou Veterinary Institute, Chinese Academy of Agriculture Sciences. The IHA method in this study is simpler and more sensitive than the enzyme-linked immunosorbent assay (ELISA) method, and referenced the same IHA kit in many previous similar experiments (Wang et al. 2012, Kang et al. 2016, Liu et al. 2018). According to the manufacturer's instructions, first prepare the IHA detection antigen, add an appropriate amount of sterile doubledistilled water $\left(\mathrm{ddH}_{2} \mathrm{O}\right)$, mix, and perform in a centrifuge at $2000 \mathrm{rpm}$ for $5 \mathrm{~min}$. Then gently aspirate the supernatant with a pipette and discard it. Add the same volume of dilution as $\mathrm{ddH}_{2} \mathrm{O}$. Shake well and put it in a $4^{\circ} \mathrm{C}$ refrigerator for about $24 \mathrm{~h}$ before using. Finally, a 96-well plate with $\mathrm{V}$ well bottom was used at the screening dilutions of 1:4, 1:16, 1:64, 1:256,

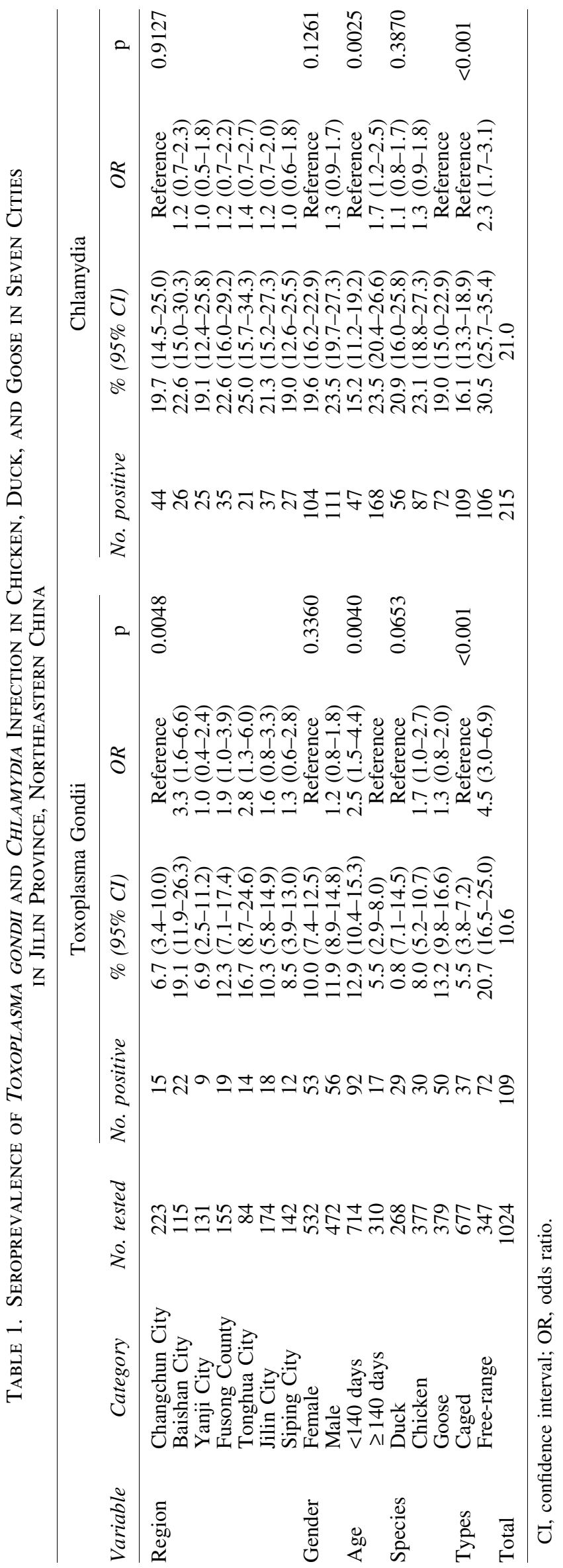


1:1024, and 1:4096. Samples reacting above 1:64 were considered positive for T. gondii and Chlamydia spp. antibodies. The sensitivity and specificity values for the testing kit used in this study have been validated by ministry of agriculture of the People's Republic of China (NY/T 5622002) (Wu et al. 2013).

\section{Statistical analysis}

Differences in the positive rates of $T$. gondii and Chlamydia serum antibodies in chickens, ducks, and geese for different variables (region, gender, age, species, and type) were analyzed by using SPSS (Release 18.0 standard version; SPSS, Inc., Chicago, IL). If the probability $p$ value was $<0.05$, the results were considered statistically significant.

\section{Results}

\section{Seroprevalence and risk factor analysis of $\mathrm{T}$. gondii}

The seroprevalence of T. gondii was $10.6 \%$ (109/1024) in all poultry, with $7.96 \%$ (30/377), $10.82 \%$ (29/268), and $13.19 \%(50 / 379)$ in chickens, ducks, and geese, respectively. T. gondii seroprevalence of poultry in different regions ranged from $8.45 \%(12 / 142)$ in Siping City to $19.13 \%(22 / 115)$ in Baishan City. Male poultry had a higher prevalence $(11.86 \%)$ than females $(9.96 \%)$. Moreover, free-range $(20.75 \%)$ poultry had a higher seroprevalence of $T$. gondii than that of caged $(5.47 \%)$. The seroprevalence in elder poultry $(12.89 \%)$ was slightly higher than that in younger ones. (5.49\%). Through the analysis of this study, free-range poultry had significantly higher seroprevalence than caged poultry $(p<0.001)$. Age was considered as a risk factor for $T$. gondii infection in rural poultry $(p<0.001)$. In addition, geography factor is a serological risk factor to Toxoplasma $(p<0.05)$ (Table 1).

\section{Seroprevalence and risk factor analysis of Chlamydia}

Of the 1024 samples detected, positive results for antiChlamydia antibodies were observed in 215 samples, the prevalence rate was $21.0 \%$. By region, positive animals were detected in each area, and Tonghua $(25.0 \%)$ had the highest positive rate, while the lowest prevalence was found in Siping City (19.01\%). Chlamydia seroprevalence in poultry of different ages ranges from $15.16 \%$ (47/310) in younger to $23.53 \%(168 / 714)$ in elder. The positive rate of Chlamydia infection in caged $(5.5 \%)$ is far lower than the free-range $(20.7 \%)$. For gender, male poultry had a slightly higher seroprevalence $(23.52 \%)$ than female poultry (19.55\%). Risk factor analysis revealed that free-range poultry had significantly higher seroprevalence than caged poultry $(p<0.001)$ and age was significantly associated with Chlamydia infection in investigated poultry $(p<0.001)$ (Table 1 ; Fig. 1).

\section{Discussion}

Rural population in China is still the majority of the total population. In recent years, the proportion of poultry farming is continuously increasing in Chinese rural areas (Liu et al. 2018). However, the prevalence of parasitic diseases in rural areas is still the main problem. Poultry are infected with $T$. gondii by eating food and water contaminated with $T$. gondii oocysts in cat feces, while humans are mainly infected by eating uncooked raw meat containing $T$. gondii tissue cysts.
Avian Chlamydia is mainly transmitted by air, and the respiratory tract is probably the most common route of transmission, followed by oral infection. The disease is already a worldwide disease with a wide range of prevalence (Zhu et al. 2008, Ni et al. 2015). Therefore, the increase in the number of poultry will increase the risk of people being infected with pathogens (Dubey 2010).

The present study also estimated the seroprevalence of $T$. gondii and Chlamydia in chicken, duck, and goose in China by systematically reviewing international and Chinese bibliographic databases. The 31 provinces of mainland China were categorized into seven regions, including central, east, north, northeast, northwest, south, and southwest of China, in our study (Table 2). The data of the seroprevalence of $T$. gondii and Chlamydia in poultry of these 31 provinces are combined with ours, indicating that $T$. gondii and Chlamydia are common prevalent in chickens, ducks, and geese in mainland China.

Toxoplasmosis is an important zoonotic disease that requires further attention (Dubey 2009). The prevalence of $T$. gondii in poultry in this study $(10.64 \%)$ was lower than those of Nanjing $(67.14 \%)$ in China (Liu et al. 2017), Hubei (24.8\%) (Luo et al. 2017), and Beijing (12.0\%) (Wen et al. 2019). In the northeast China, our results are lower than that in chickens (37.5\%) (Zhu et al. 2008). However, the positive rate in present studies higher than that of West Africa (7.67\%) (Sarr et al. 2020), Jilin $(5.15 \%)$, and Liaoning $(5.56 \%)$. A possible explanation is that poultry have different exposures to pathogens in different areas. Other reasons for the different prevalence rates in various regions include the climate, altitude, breeding density, and animal welfare of each region. In addition, younger poultry are exposed to the environment for less time than the older, so the chance of infection with $T$. gondii is relatively low (Table 1). Data from this study indicate that the seroprevalence of Toxoplasma in free-range poultry increases with age, which is consistent with the previous studies (Bai et al. 2017). Probably because that $T$. gondii is an opportunistic parasite, and the accumulation of $T$. gondii may run through its life.

Chlamydia is an obligate intracellular gram-negative bacterium, which is widely distributed throughout the world and endangers human health and has a huge economic impact on animal husbandry (Cong et al. 2014, Cechova et al. 2018). In this study, the overall seropositivity rate for Chlamydia $(21 \%)$ exposure in poultry (Table 1) is higher than those in Slovakia (6.88\%) (Cechova et al. 2018), 15.0\% in chickens in Italy (Donati et al. 2018), and 7.3\% in wild birds in Poland (Krawiec et al. 2015). However, the rate detected in our study was lower than the rate of $39.67 \%$ reported in China (Nie et al. 2018). Differences in Chlamydia seroprevalence probably depend on immunity, feeding levels and stress, livestock practices, and animal welfare. Besides, the high seroprevalence of Chlamydia in poultry in this study indicates that there are risks of zoonotic disease in northern China.

In the research, there was a significant difference between the caged and the free-range poultry in the prevalence of $T$. gondii and Chlamydia. Similarly, elder poultry also have significant difference with young poultry. Reason was taken into account that elder and free-range poultry have more range of activities and larger space to find food than younger and caged poultry, so there was more opportunity to access the oocysts of these pathogens. Therefore, ages and type were taken into consideration as risk factors. We also found that 


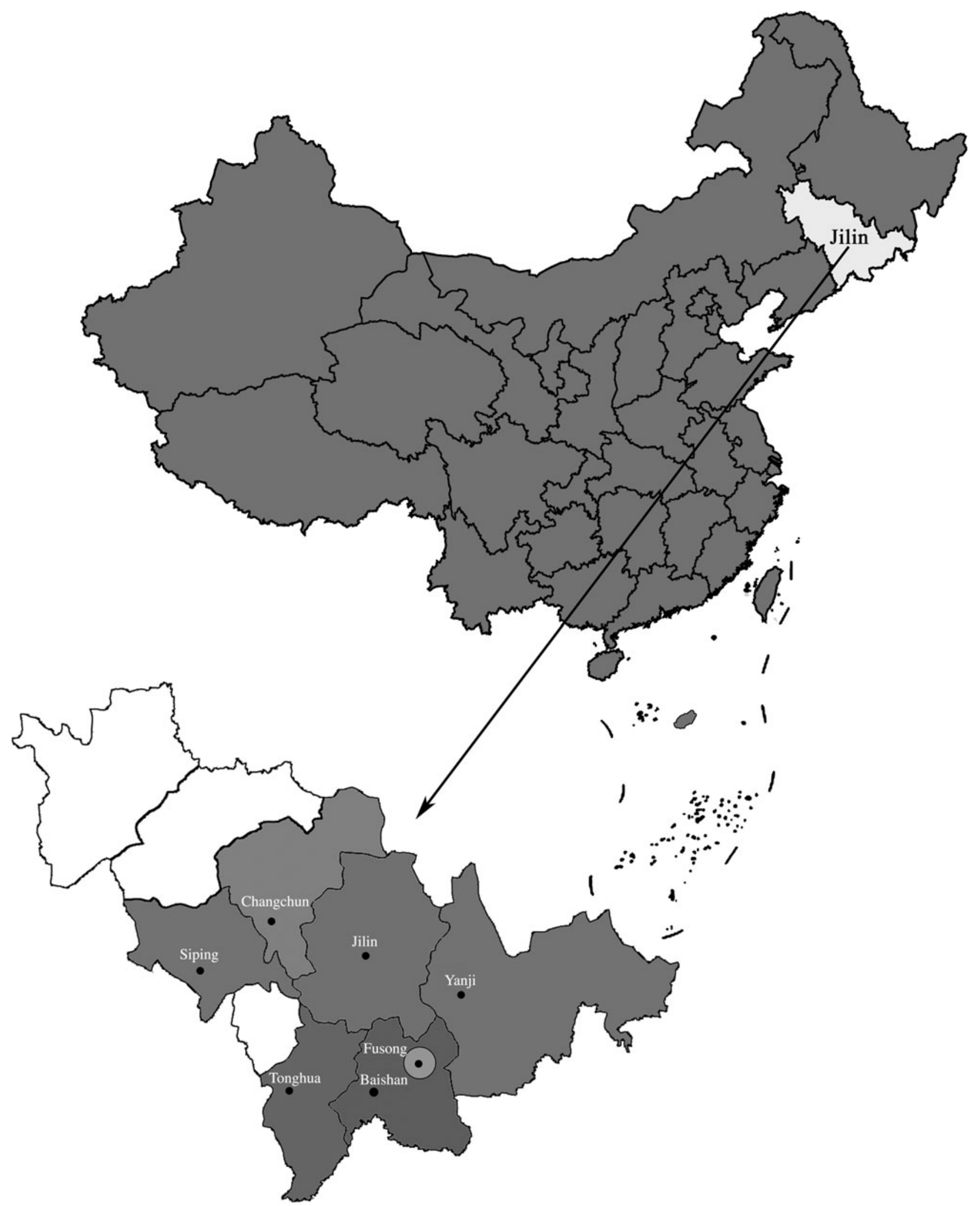

FIG. 1. Geographic distribution of the regions in northeast China where poultry sampling was performed. 


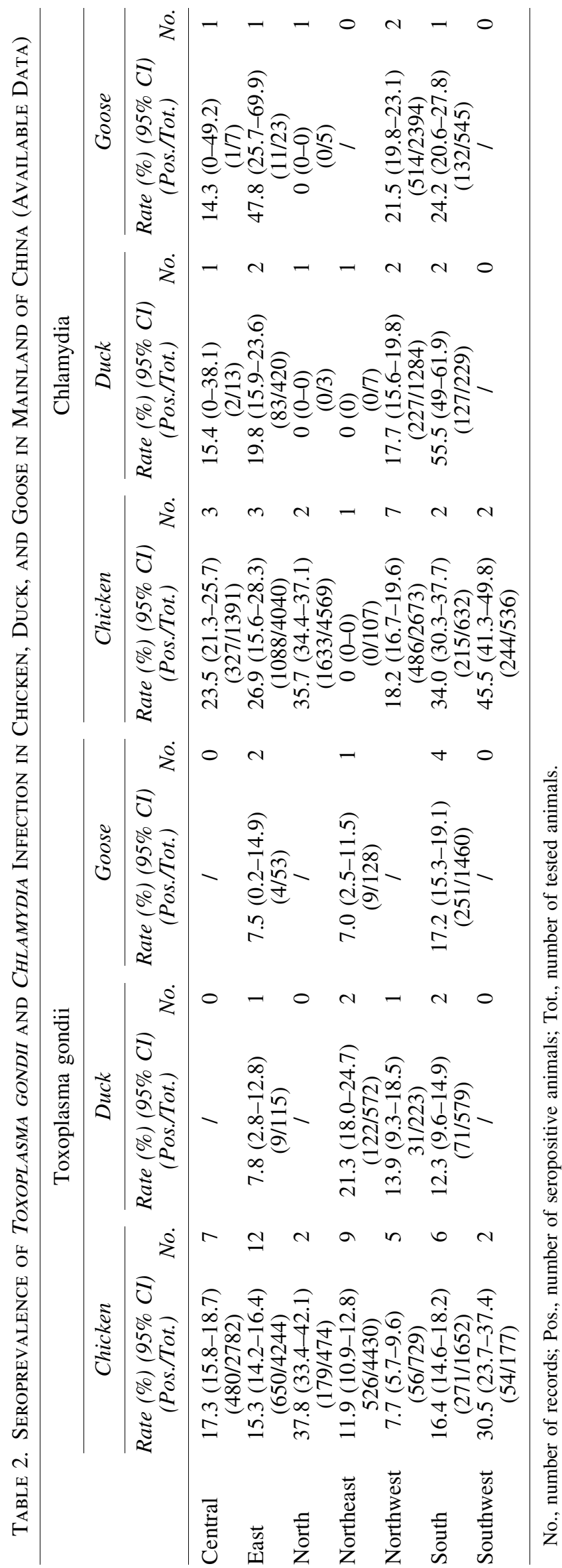

the geography was considered a risk factor for $T$. gondii. Statistics show that Baishan and Tonghua cities have the highest seroprevalence for both pathogens. It may be related to the serious industrial pollution in these two cities, resulting in a harsh environment, and the poor farming conditions of the two sampled farms. Moreover, our results show that there was no significant difference in the seropositive rates of Chlamydia and $T$. gondii between gender and species $(p>0.05)$. The seroprevalence of Chlamydia was statistically similar indicating that pathogenic bacteria may be transmitted in poultry mainly through direct contact and were less affected by environmental changes. In recent years, as the number of poultry farming has increased, protecting poultry from infection by pathogens has become a top priority and preventive measures should be strengthened.

According to our knowledge, our study was the first investigation of the seroprevalence of $T$. gondii and Chlamydia in chickens, ducks, and geese in rural areas of northeast China. A total of 1024 poultry blood samples were analyzed by using IHA. More than a quarter of the poultry are positive for pathogens, indicating that they are highly distributed in the rural areas of seven cities in Jilin province in northeastern China and may require precautionary measures such as monitoring cat infections. Risk factor analysis revealed that age and type of poultry were significantly associated with these two pathogens infection in investigated poultry. Moreover, regional factors are unique risk factors for $T$. gondii. This study provides a lot of new data on the epidemiology of zoonotic parasitic diseases in poultry in northeastern China, and it is more convenient to further study the prevalence and distribution of these pathogens.

\section{Author Disclosure Statement}

No conflicting financial interests exist.

\section{Funding Information}

This work was supported by the Key Scientific and Technological Achievements Transformation Project of Jilin Province (project no. 20170307016NY).

\section{References}

Bai MJ, Zou Y, Elsheikha HM, Ma JG, et al. Toxoplasma gondii infection in farmed wild boars (Sus scrofa) in three cities of Northeast China. Foodborne Pathog Dis 2017; 14:379-385.

Cechova L, Halanova M, Babinska I, Danisova O, et al. Chlamydiosis in farmed chickens in Slovakia and zoonotic risk for humans. Ann Agric Environ Med 2018; 25:320-325.

Cong W, Huang SY, Zhang XX, Zhou DH, et al. Chlamydia psittaci exposure in pet birds. J Med Microbiol 2014; 63:578-581.

Donati M, Laroucau K, Guerrini A, Balboni A, et al. Chlamydiosis in backyard chickens (Gallus gallus) in Italy. Vector Borne Zoonotic Dis 2018; 18:222-225.

Dubey JP. History of the discovery of the life cycle of Toxoplasma gondii. Int J Parasitol 2009; 39:877-882.

Dubey JP. Toxoplasma gondii infections in chickens (Gallus domesticus): Prevalence, clinical disease, diagnosis and public health significance. Zoonoses Public Health 2010; 57:60-73.

Elmore SA, Jones JL, Conrad PA, Patton S, et al. Toxoplasma gondii: Epidemiology, feline clinical aspects, and prevention. Trends Parasitol 2010; 26:190-196. 
Hill DE, Dubey JP. Toxoplasma gondii as a parasite in food: Analysis and control. Microbiol Spectr 2016; 4:1-17.

Jones JL, Lopez A, Wilson M, Schulkin J, et al. Congenital toxoplasmosis: A review. Obstet Gynecol Surv 2001; 56:296-305.

Kang YH, Cong W, Qin SY, Shan XF, et al. First report of Toxoplasma gondii, Dirofilaria immitis, and Chlamydia felis infection in stray and companion cats in Northeastern and Eastern China. Vector Borne Zoonotic Dis 2016; 16:654-658.

Krawiec M, Piasecki T, Wieliczko A. Prevalence of Chlamydia psittaci and other Chlamydia species in wild birds in Poland. Vector Borne Zoonotic Dis 2015; 15:652-655.

Li J, Guo W, Kaltenboeck B, Sachse K, et al. Chlamydia pecorum is the endemic intestinal species in cattle while $C$. gallinacea, $C$. psittaci and $C$. pneumoniae associate with sporadic systemic infection. Vet Microbiol 2016; 193:93-99.

Li L, Luther M, Macklin K, Pugh D, et al. Chlamydia gallinacea: A widespread emerging Chlamydia agent with zoonotic potential in backyard poultry. Epidemiol Infect 2017; 145:2701-2703.

Liu F, Li JM, Zeng FL, Zong Y, et al. Prevalence and risk factors of brucellosis, chlamydiosis, and bluetongue among sika deer in Jilin Province in China. Vector Borne Zoonotic Dis 2018; 18:226-230.

Liu XC, He Y, Han DG, Zhang ZC, et al. Detection of Toxoplasma gondii in chicken and soil of chicken farms in Nanjing region, China. Infect Dis Poverty 2017; 6:62.

Luo H, Li K, Shahzad M, Zhang H, et al. Seroprevalence of Toxoplasma gondii infection in wild boars, wild rabbits, and wild chickens in Hubei Province, China. Korean J Parasitol 2017; 55:85-88.

Ni X, Qin S, Lou Z, Ning H, et al. Seroprevalence and risk factors of Chlamydia infection in domestic rabbits (Oryctolagus cuniculus) in China. Biomed Res Int 2015; 2015:460473.

Nie LB, Liang QL, Zou Y, Gao YH, et al. First report of Chlamydia seroprevalence in farmed wild boars in China. Vector Borne Zoonotic Dis 2018; 18:504-508.

Qin SY, Yin MY, Cong W, Zhou DH, et al. Seroprevalence and risk factors of Chlamydia abortus infection in Tibetan sheep in Gansu province, northwest China. Sci World J 2014; 2014: 193464.

Sachse K, Bavoil PM, Kaltenboeck B, Stephens RS, et al. Emendation of the family Chlamydiaceae: Proposal of a single genus, Chlamydia, to include all currently recognized species. Syst Appl Microbiol 2015; 38:99-103.

Sarr A, Galal L, Boumediene F, Hamidovic A, et al. Seroprevalence and risk factors of Toxoplasma gondii infection in free-range chickens in Senegal, West Africa. Vector Borne Zoonotic Dis 2020; 20:15-21.

Wang M, Wang YH, Ye Q, Meng P, et al. Serological survey of Toxoplasma gondii in Tibetan mastiffs (Canis lupus familiaris) and yaks (Bos grunniens) in Qinghai, China. Parasit Vectors 2012; 5:35.

Weiss LM, Dubey JP. Toxoplasmosis: A history of clinical observations. Int J Parasitol 2009; 39:895-901.

Wen J, Zou J, Huang X, Wen H, et al. Identification of candidate antigens by 2-DE Immunoblotting for diagnosis of Toxoplasma gondii infection in chickens and rabbits. Exp Parasitol 2019; 204:107723.

Wu SM, Huang SY, Xu MJ, Zhou DH, et al. Chlamydia felis exposure in companion dogs and cats in Lanzhou, China: A public health concern. BMC Vet Res 2013; 9:104.

Zhang XX, Cai YN, Wang CF, Jiang J, et al. Seroprevalence and risk factors of Toxoplasma gondii infection in stray dogs in northern China. Parasitol Res 2015; 114:4725-4729.

Zhang XX, Qin SY, Li X, Ren WX, et al. Seroprevalence and related factors of Toxoplasma gondii in pigeons intended for human consumption in Northern China. Vector Borne Zoonotic Dis 2019; 19:302-305.

Zhu J, Yin J, Xiao Y, Jiang N, et al. A sero-epidemiological survey of Toxoplasma gondii infection in free-range and caged chickens in northeast China. Vet Parasitol 2008; 158:360-363.

Address correspondence to: Quan Zhao

Department of Parasitology

College of Animal Science and Technology Jilin Agricultural University

Changchun, Jilin 130118

P.R. China

E-mail: zhaoquan0825@163.com

Jing Jiang

Department of Parasitology College of Life Science

Changchun Sci-Tech University Shuangyang, Jilin 130600

P.R. China

E-mail: jiangjingxiaoyao@163.com 